\title{
Preliminary observations of brittle compressive failure of columnar saline ice under triaxial loading
}

\author{
E. T. GRatz and E. M. Schulson \\ Thayer School of Engineering, Dartmouth College, Hanover, NH 03755, U.S.A.
}

\begin{abstract}
Experiments were performed on columnar saline ice prepared in the laboratory. Specifically, using a true multiaxial loading system, relatively large cubes ( $159 \mathrm{~mm}$ on edge) were proportionally loaded along three orthogonal directions; viz. perpendicular to $\left(\sigma_{11}\right.$ and $\left.\sigma_{22}\right)$ and parallel to $\left(\sigma_{33}\right)$, the long axis of the columnar grains. The ice was strained at $10^{-2} \mathrm{~s}^{-1}$ at $-10^{\circ} \mathrm{C}$ where it behaved in a brittle manner. Four sets of experiments were performed by setting the ratio $\sigma_{22} / \sigma_{11}$ to $0,0.25,0.5$ or 1 , and varying the ratio $\sigma_{33} / \sigma_{11}$ and $\sigma_{11} / \sigma_{33}$. Sections through the failure surface were then constructed. For loading along $\sigma_{22}=0$, the confining stress did not significantly affect the maximum principal stress at failure. For loading along $\sigma_{22}=0.25 \sigma_{11}$, $\sigma_{22}=0.5 \sigma_{11}$ and $\sigma_{22}=\sigma_{11}$, the application of a moderate confining stress resulted in a large increase in failure stress. However, further increase in the level of the confining stress did not have a significant effect on the failure stress. The observations are presented in terms of a failure surface and are discussed in terms of the possible failure mechanisms.
\end{abstract}

\section{INTRODUCTION}

An important problem in ice mechanics is the compressive failure of columnar ice under multiaxial loading. The contact zone, for instance, between a sheet of first-year sea ice and an obstacle, such as an ice floe or an engineered structure, is characterized by a multiaxial state of compressive stress. Predictions of ice forces, therefore, require a good understanding of the failure processes under complex stress states. To this end, a study has been initiated to examine systematically this kind of failure in columnar saline ice. The ultimate objectives are to establish the complete three-dimensional failure surfaces and to understand the underlying failure mechanisms. This paper presents some first observations. The focus is on brittle failure; i.e. on the pseudo-elastic kind of failure which occurs when ice is rapidly loaded.

To our knowledge this is the first report on the behavior of columnar saline ice under proportional triaxial loading within the brittle regime. Earlier work by Häusler (1982) on similar material explored the ductile regime, and a more recent study by RichterMenge (1991) on columnar first-year sea ice with preferential orientation of the $c$-axes entered the brittle regime only for uniaxial tests. Also tests by Timco and Frederking (1986) on columnar sea ice entered the brittle regime only for uniaxial loading along the columns. Triaxial tests by Sammonds and others (1989), although within the brittle regime, were performed on multi-year sea ice of complex structure, and experiments by Jones (1982) were conducted on granular fresh-water ice under constant confining pressures.

\section{EXPERIMENTAL}

\section{Growth procedure}

Pucks of laboratory-grown saline ice (LGSI) were grown in a 8171 plastic tank using a procedure of unidirectional cooling described in Kuehn and others (1990). A solution of saline water was prepared by mixing the commercial product "Instant Ocean" with hot, filtered Hanover tap water. The salinity of the solution was about $23 \%$. The solution was then equilibrated to $0^{\circ} \mathrm{C}$ before a cooling plate was placed on the surface of the solution and the sides of the tank insulated. The temperature of the cooling plate was set at $-30^{\circ} \mathrm{C}$ which resulted in $300 \mathrm{~mm}$ thick pucks after about two weeks of growth.

\section{Ice structure}

The structure of LGSI is characterized by columnar grains whose long axes are parallel to the direction of heat flow during the growth process. The $c$-axes were randomly orientated within the horizontal plane of the puck and were contained within $\pm 15^{\circ}$ of this plane. The average equivalent diameter of the columnar grains was $4.7 \mathrm{~mm}$ and was obtained as described by Kuehn and Schulson (1994). Platelet-like arrays of brine pockets existed within individual grains, and irregular brine channels were observed at some boundaries between columns. Salinities ranged from 3.9-4.4\%o. Figure 1 shows thin sections taken parallel to and perpendicular to the columns. 

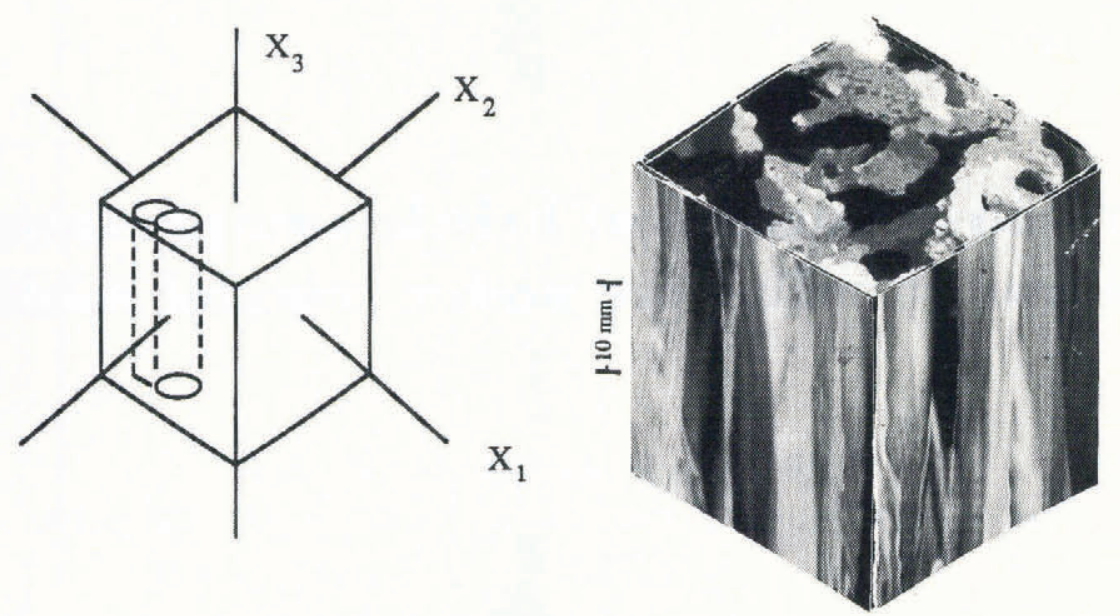

Fig. 1. Schematic sketch of LGSI structure and composite photograph of structure as viewed using thin sections between crosspolarized filters.

\section{Sample preparation and dimensions}

Cubic-shaped samples $159 \times 159 \times 159 \mathrm{~mm}^{3}$ were cut from the pucks. They were milled to a tolerance of $0.1 \mathrm{~mm}$ across the face of $159 \mathrm{~mm}$; i.e. to a parallelism of $6.3 \times$ $10^{-4}$ radians. The samples were placed in insulated boxes after milling. If they were to be used immediately,then they were equilibrated at the testing temperature of $-10^{\circ} \mathrm{C}$; otherwise they were stored at $-40^{\circ} \mathrm{C}$. All stored samples were equilibrated at the testing temperature for a minimum of 48 hours prior to testing, a time based on equilibrium tests on cubic samples with a side dimension of $214 \mathrm{~mm}$.

\section{Testing procedures}

Testing was performed in the Ice Research Laboratory (IRL) using a Multiaxial Testing System housed within a cold room. This device allows for proportional loading along three orthogonal directions and was specially built for the IRL by MTS Corp. Two loading actuators on each of the three axes maintained the centroid of the sample in a fixed position during loading. Output from the testing machine was recorded using a multi-channel data acquisition system. Data included output from load transducers associated with each actuator and from six extensometers mounted on metallic-brush loading platens. The maximum principal load was applied using the stroke control mode of the testing machine, where the stroke rate was calculated to correspond to the target strain rate of $1 \times 10^{-2} \mathrm{~s}^{-1}$. Confining loads were applied in proportion to the master load to simulate natural loading scenarios.

\section{Loading paths}

Figure 2 shows the reference co-ordinate system and the loading paths. The axes $X_{1}$ and $X_{2}$ were orthogonal to the columns and $X_{3}$ was parallel to the columns. Tests were conducted along planes of constant ratio $\sigma_{22} / \sigma_{11}$ in $\sigma_{11}-\sigma_{22}-\sigma_{33}$ stress space, where $\sigma_{11}, \sigma_{22}$, and $\sigma_{33}$ are the principal stresses. The loading paths were $\sigma_{22} / \sigma_{11}=0$, $0.25,0.5$ and 1 , along which $\sigma_{33}$ was varied. In some tests $\sigma_{11}$ was the maximum principal stress, while in others $\sigma_{33}$ was the maximum principal stress. Compressive stresses were taken to be positive.

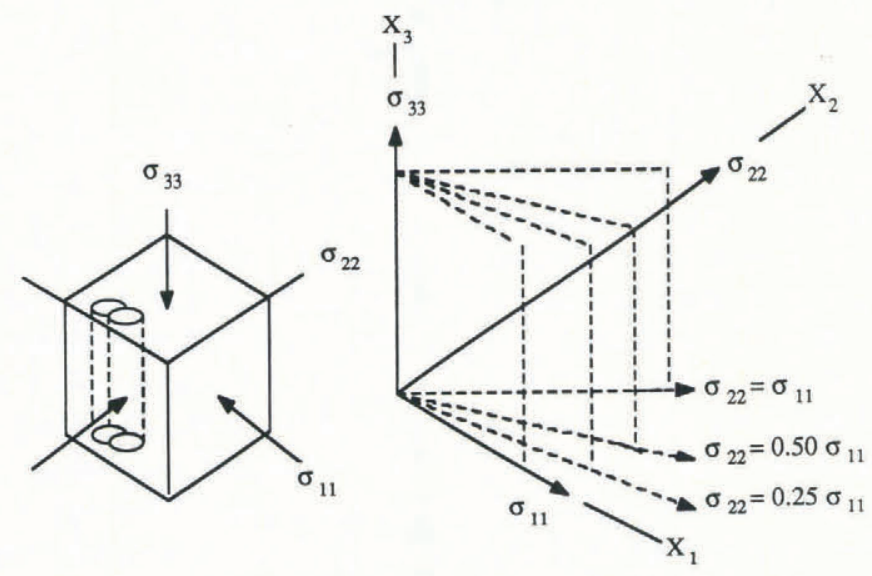

Fig. 2. Schematic sketch of loading stresses and the loading paths examined in the all-compressive octant. 


\section{RESULTS}

Failure occurred in a brittle manner for all tests. The deformation was characterized by essentially linear stress strain behavior and by the collapse of the specimen after about $0.20 .5 \%$ shortening along the master loading direction. The failure strains along the master axis increased as the intermediate principal stress approached the maximum principal stress. Once all three orthogonal strains were compressive, the strain corresponding to the maximum principal stress either decreased or increased only slightly as the intermediate stress approached the maximum stress. Figure 3 shows stress-strain curves from one of the tests for the ratio $\sigma_{11}: \sigma_{22}: \sigma_{33}=2: 1: 4$. Generally the failure stresses were scattered, as will become apparent. Yet within the scatter important trends could be seen.

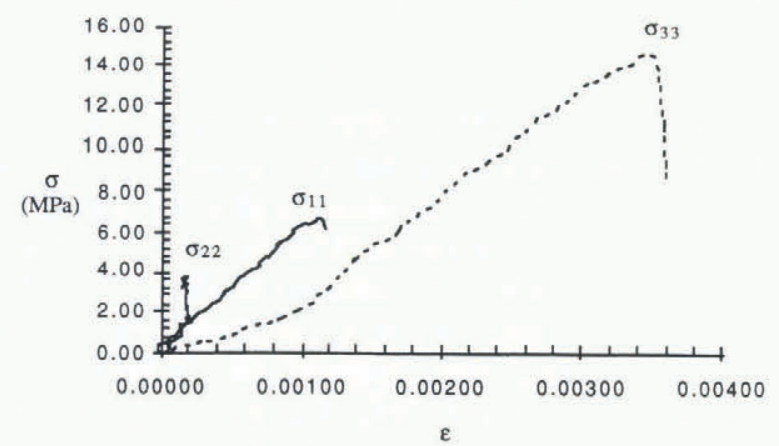

Fig. 3. Stress-strain curves for a lest conducted where $\sigma_{33}$ was the maximum principal stress, and the ratio $\sigma_{11}: \sigma_{22}: \sigma_{33}$ was $2: 1: 4$. The strain, $\epsilon$, corresponds to $\epsilon_{11}$ for $\sigma_{11}, \epsilon_{22}$ for $\sigma_{22}$ and $\epsilon_{33}$ for $\sigma_{33}$. The strain rate along $X_{3}$ was $10^{-2} s^{-1}$, and the lest was conducted at $T=-10^{\circ} \mathrm{C}$.

\section{Path $\left(\sigma_{11}, \mathbf{0}, \sigma_{33}\right)$}

Figure 4 shows the failure envelope for biaxial loading along and across the columns. The results fall within a region roughly delineated by the uniaxial failure strengths for loading along the columns, $\sigma_{33 . f}=10.8 \pm 1.6 \mathrm{MPa}$, and loading across the columns, $\sigma_{11, \mathrm{f}}=3.74 \pm 0.50 \mathrm{MPa}$ (Kuehn and Schulson, 1994; Smith and Schulson, 1994). With the exception of one point, the uniaxial data along the columns were obtained by Kuehn and Schulson (1994), and are shown as open squares; uniaxial data across the columns were obtained by Smith and Schulson (1994) and are shown as crosses. The data show no indication that the addition of a confining stress increased the maximum principal failure stress.

In these tests failure occurred by splitting along the columns. Uniaxial loading across and along the columns resulted in planar cracks perpendicular to $X_{2}$ (Fig. 5a) and in column-like shards parallel to the loading direction (Fig. $5 \mathrm{c}$ ), respectively. Biaxial loading generally led to planar cracks perpendicular to the direction of no load (Fig. 5b).

Path $\left(\sigma_{11}, \sigma_{11}, \sigma_{33}\right)$

Figure 6 shows the failure envelope for triaxial loading when $\sigma_{22}=\sigma_{11}$. The results are plotted as the projection

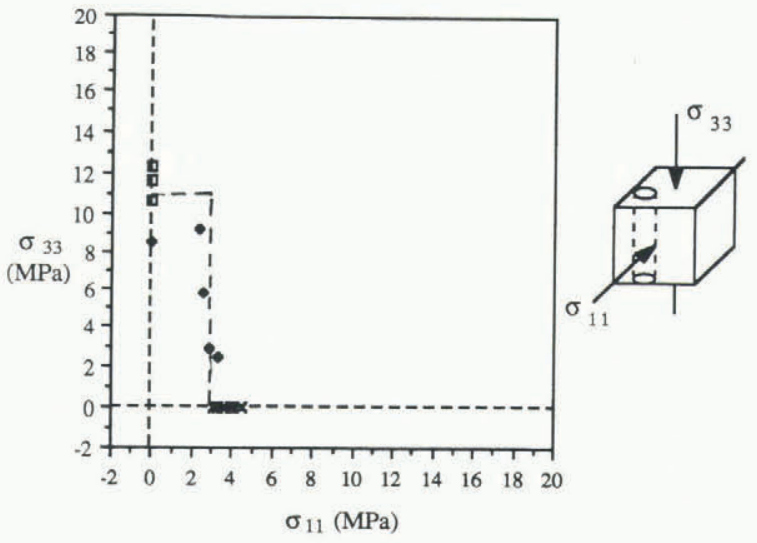

Fig. 4. The failure surface on the plane $\sigma_{11}-\sigma_{33}$ for loading $\sigma_{22}=0$. Uniaxial results of Kuehn and Schulson (1994) are denoted by squares and biaxial results of Smith and Schulson (1994) are represented by crosses. Tests were conducted at a strain rate of $10^{2} \mathrm{~s}^{-1}$ at $T=-10^{\circ} \mathrm{C}$.

onto the $\sigma_{11}-\sigma_{33}$ plane. Starting from the conditions of biaxial confinement, the maximum principal failure stress increased from about $9 \mathrm{MPa}$ for $\sigma_{33}=0$ to about $14 \mathrm{MPa}$ for $\sigma_{33}=0.5 \sigma_{11}$. With additional loading along the columns, the failure stress appeared not to change significantly, even as the stress state approached pure hydrostatic conditions. Similar behavior was noted when starting from uniaxial loading along the columns. The maximum principal stress at failure, in this case $\sigma_{33}$, increased from a uniaxial value of about $10.8 \mathrm{MPa}$ for $\sigma_{11}=\sigma_{22}=0$ to about $16 \mathrm{MPa}$ for triaxial loading where $\sigma_{11}=\sigma_{22}=0.25 \sigma_{33}$. As the ratio $\sigma_{11} / \sigma_{33}$ was increased further, again approaching hydrostatic conditions, the stress at failure appeared not to show significant additional variation.

The failure modes in these cases are more complicated. Betweeen $\sigma_{33}=0$ and $\sigma_{33}=0.5 \sigma_{11}$, the mode changed from splitting across the columns, described by Smith and Schulson (1993) as spallation out of the major loading plane, to a kind of crushing/cracking localized near the surface. This surface cracking was most concentrated at one of the surfaces orthogonal to the maximum applied load. It was also the failure mode observed under higher confinement for tests where $\sigma_{33}$ was a greater fraction of $\sigma_{11}$. When $\sigma_{33}>\sigma_{11}$, including the case $\sigma_{11}=0.25 \sigma_{33}$, failure again occurred near the surface. Tests were not run at lower confinements, except for the case of zero confinement (uniaxial loading along the columns) where, as noted above, the failure was characterized by splitting

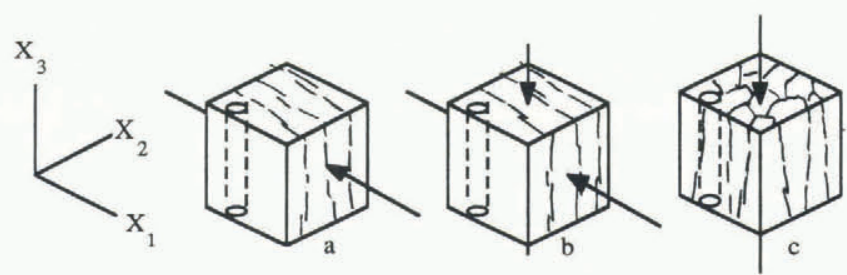

Fig. 5. Schematic sketches of failure modes for loading $\sigma_{22}=0$. 


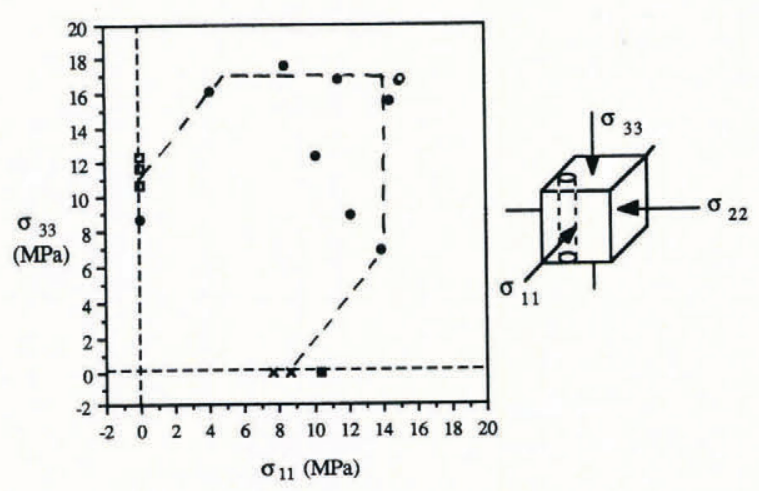

Fig. 6. Projection on to the $\sigma_{11}-\sigma_{33}$ plane of the failure surface for loading $\sigma_{22}=\sigma_{11}$. Overlapping open circles in upper right are for tests conducted using both greased and ungreased brass-plate loading surfaces. Tests were conducted at a strain rate of $10^{-2} \mathrm{~s}^{-1}$ at $\mathrm{T}=-10^{\circ} \mathrm{C}$.

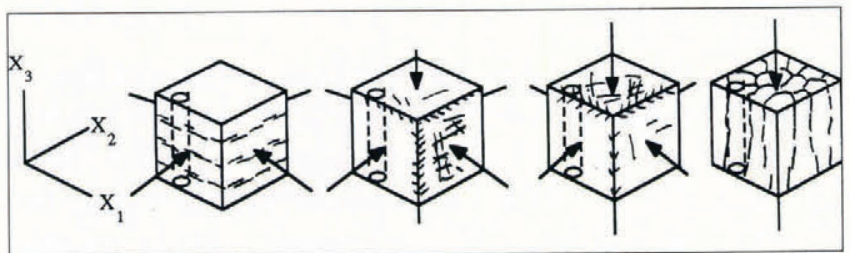

Fig. 7. Schematic sketches of failure modes for loading $\sigma_{22}=\sigma_{11}$.

along the columns. Figure 7 shows a schematic sketch of these modes.

Path $\left(\sigma_{11}, 0.5 \sigma_{11}, \sigma_{33}\right)$

Figure 8 shows the failure envelope for triaxial loading when $\sigma_{22}=0.5 \sigma_{11}$. Similar behavior is apparent. Again a large increase in failure stress was seen upon adding either a moderate stress along the columns to a predominantly across-column loading scenario or a moderate confinement across the columns to a predominantly alongcolumn loading scenario. Further increases in the confining stresses did not significantly affect the maximum principal failure stress. For $\sigma_{11}>\sigma_{33}$ failure occurred by spalling across the columns when $\sigma_{33}$ was zero, but again became localized near the surface of the specimen when $\sigma_{33}$ was increased. For $\sigma_{33}>\sigma_{11}$ surface failure occurred for all cases studied along the path $\sigma_{22}=$ $0.5 \sigma_{11}$.

\section{Path $\left(\sigma_{11}, 0.25 \sigma_{11}, \sigma_{33}\right)$}

Figure 9 shows the failure envelope for biaxial loading when $\sigma_{22}=0.25 \sigma_{11}$. Still again similar trends are evident within the scatter, and the failure modes were the same as under the other cases of triaxial loading.

\section{Summary}

The results for constant ratios of $\sigma_{22} / \sigma_{11}$ can be summarized as follows. Starting from the conditions of biaxial loading across the columns, the stress at failure

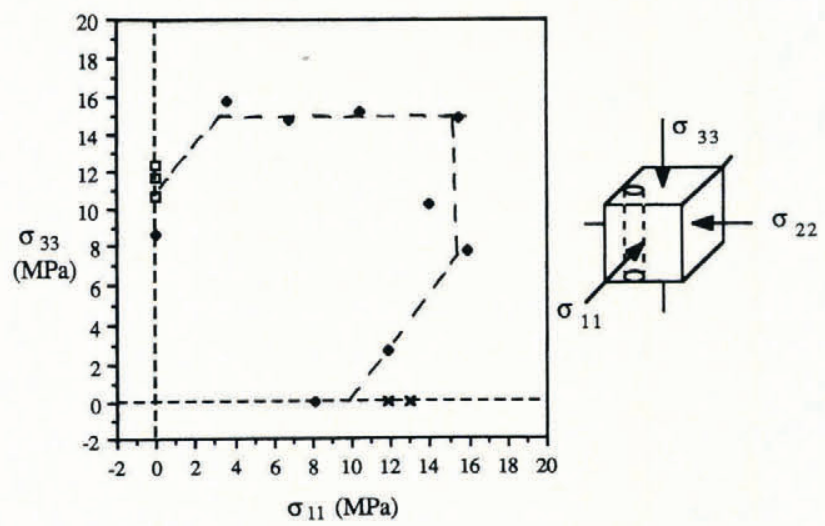

Fig. 8. Projection on to the $\sigma_{11}-\sigma_{33}$ plane of the failure surface for loading $\sigma_{22}=0.5 \sigma_{11}$. Tests were conducted at a strain rate of $10^{-2} \mathrm{~s}^{-1}$ at $\mathrm{T}=-10^{\circ} \mathrm{C}$.

increased by about $50 \%$ upon the application of a moderate confining load along the columns. Upon the application of a greater confining load little systematic change in strength was noted. The trends are repeated starting from conditions of uniaxial loading along the columns. Upon application of a moderate biaxial confinement across the columns, the failure stress increased again by about $50 \%$, but changed little in a systematic manner upon further increase in the confinement. In other words, a low level of triaxial confinement resulted in a large increase in the failure stress; further increase in the confinement had no significant additional effect on the failure stress.

\section{Failure surface}

An approximate shape of the brittle compressive failure surface was obtained as follows: the points on the failure envelopes were first described by a series of straight lines (as shown in Figs 4, 6, 8 and 9). These lines were then plotted in principal stress space and connected to form planes. The planes then formed the boundaries on the failure surface. Figure 10 shows the result. Two views are given to aid visualization. Starting from the origin, the

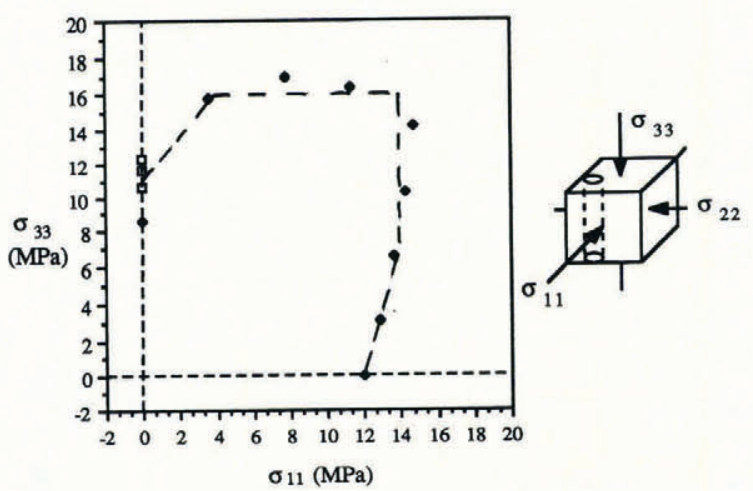

Fig. 9. Projection on to the $\sigma_{11}-\sigma_{33}$ plane of the failure surface for loading $\sigma_{22}=0.25 \sigma_{11}$. Tests were conducted at a strain rate of $10^{-2} \mathrm{~s}^{-1}$ at $-10^{\circ} \mathrm{C}$. 

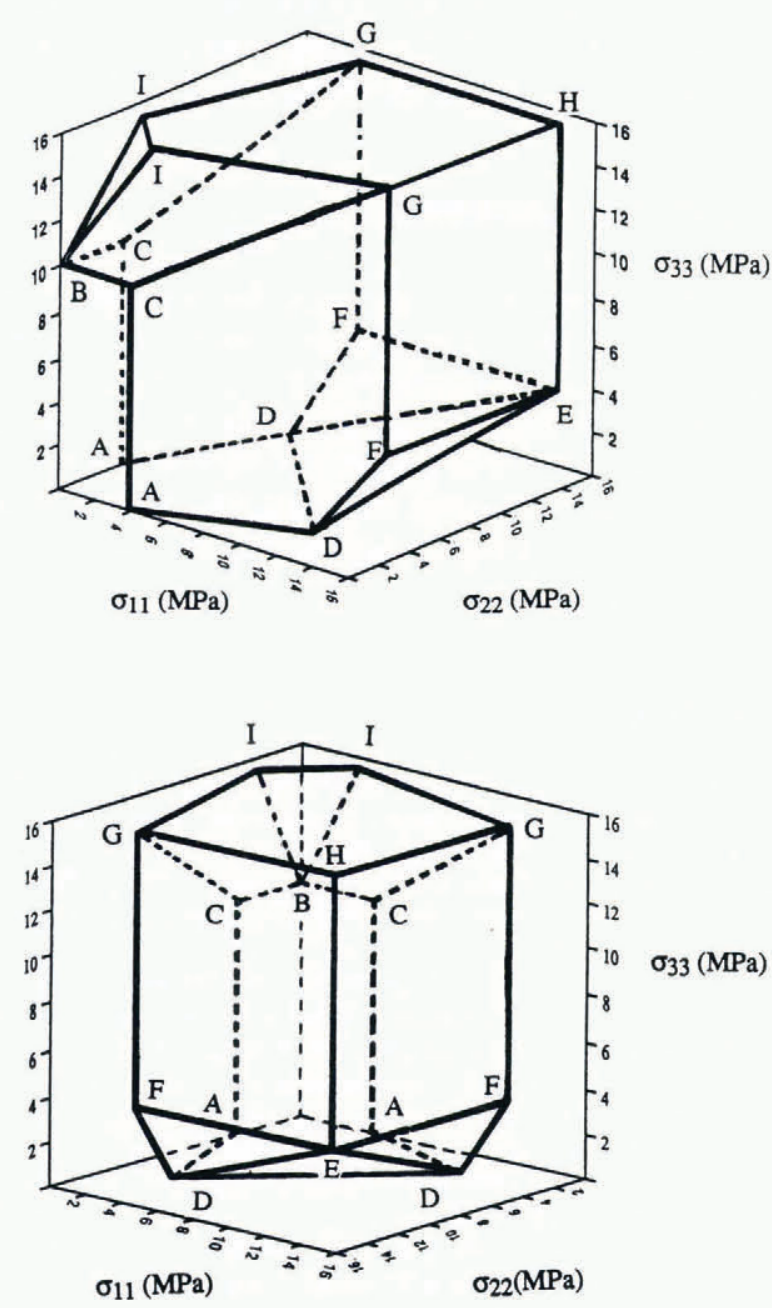

Fig. 10. Preliminary brittle compressive failure surface for saline, columnar ice loaded at a strain rate of $10^{-2} \mathrm{~s}^{-1}$, at $T=-10^{\circ} \mathrm{C}$.

surface first expands about the hydrostatic line. The surface then appears to contract about the hydrostatic line as the hydrostatic component of the stress state increases further.

The real surface will not have sharp corners and edges. Instead, more rounded extremities are expected. Nevertheless, Figure 10 gives the first experimental-based indication of the brittle compressive failure surface for ice. It also shows how the failure strength increases rapidly from relatively low values under uniaxial loading to quite high values under triaxial loading.

\section{DISGUSSION}

It is important to note again that the failure surface is only a first approximation. More experiments are needed to define it more clearly. Nevertheless, at this juncture, the picture begins to emerge, albeit tentatively.

The stress state, and particularly the hydrostatic component, seems to have a much greater effect on the brittle compressive strength of ice than it does, say, on the yield strength of a ductile material like aluminium.
In the latter case, failure (i.e. yielding) is governed by crystallographic slip, and that process is driven solely by the deviatoric part of the stress tensor. As a result, the attendant failure surface for isotropic material, for instance, can be described as a tube of constant diameter whose axis lies along the hydrostatic loading path, $\sigma_{11}=\sigma_{22}=\sigma_{33}$, and whose diameter is set by the critical shear stress for slip. Since the critical shear stress is independent of the stress normal to the slip plane, the hydrostatic component of the stress state plays no role in determining how near to failure (i.e. to yielding) is a multiaxially loaded ductile material. In fact, a ductile material loaded purely hydrostatically should not fail, regardless of how high the stress is. In brittle ice the situation is different. The failure surface indicates that the hydrostatic component impedes failure up to a point; and then, if great enough, it appears to cause failure. The last point is unusual, even though it was noted by Häusler (1982) who also used metallic-brush platens to study ice, but within the ductile regime. Yet, barring this point, it is clear that different failure mechanisms are operating in the ice. Most probably, they are based upon the nucleation, growth and interaction of cracks.

One mechanism is the frictional crack-sliding/wingcrack mechanism (Nemat-Nasser and Horii, 1982; Ashby and Hallam, 1986). Its operation in both fresh-water and salt-water columnar ice was reported elsewhere Cannon and others, 1990; Schulson and others, 1991; Smith and Schulson, 1993, 1994). The wing-crack mechanism is based upon the opening up of wing cracks at the tips of sliding parent cracks which nucleate at grain boundaries. The hydrostatic part of the stress tensor is important because it affects the stress normal to the plane of sliding and hence the frictional resistance to sliding: when too great, the hydrostatic stress suppresses sliding altogether. The wing-crack mechanism, therefore, is limited to rather low degrees of hydrostatic stress given the relatively high friction coefficient of ice on ice (Jones and others, 1991). Point $\mathrm{A}$ and lines $\mathrm{AD}$ and $\mathrm{AC}$ on the failure surface can be explained in this way.

Under higher degrees of confinement different mechanisms operate. For instance, under higher levels of biaxial loading across the columns $\left(\sigma_{33}=0\right)$ ice fails by spalling out of the loading plane. This can be explained in terms of the Hertzian contact mechanism described by Smith and Schulson (1993). Line DD on the failure surface is rationalized in this way. So, too, could be plane DFE because a small stress along the columns is expected not to suppress spalling but only to increase the failure stress required for this process.

What cannot be explained on the basis of either wing crack or Hertzian mechanics is the failure which occurs under higher confinement. This corresponds to the loading paths which intersect the larger facets of the failure surface, EFGH and GHI. Here, a third mechanism operates. Its nature remains to be elucidated. What is clear is that this new mechanism does not involve the growth of cracks because the far-field compressive stress field prevents growth. Instead, it may involve the development of a critical crack density which is a problem in crack nucleation and crack interaction. 
The third mechanism does not appear to be a result of the metallic-brush platens. From additional tests conducted using loading platens made from solid brass plates there was little difference in either the loads at failure or the failure mode when loading along the hydrostatic when compared to the results using brush platens (personal communication from E.T. Gratz). Nor was there a difference when a layer of grease separated the ice from the solid brass plates. For both of these cases, the failure stresses were close to those for samples loaded using the brush platens, as shown in Figure 6 . For both cases failure again was confined to the surface of the samples, as observed for the tests conducted using the brush platens. Thus, based on these results, it appears that the failure mode under higher triaxial confinement is not an artifact of the brush platens. Instead, failure under near-hydrostatic loading is attributed to the stress-free sample edges, a characteristic of the loading system. In keeping with this view is the additional observation (personal communication from E. T. Gratz) that pore-free columnar ice (i.e. freshwater ice) also fails near the surface when loaded hydrostatically, implying that pore collapse cannot account for such failure of the saline ice.

Thus, it appears that several mechanisms contribute to the brittle compressive failure of columnar saline ice. The process is more complicated than envisaged by Hallam (1986) who, when considering the failure of isotropic granular ice, modeled it in terms of the frictional crack-sliding/wing-crack mechanism only. Mechanisms are specific to particular regions of principal stress space and thus dictate different failure criteria depending upon the stress state. It also appears that under higher degrees of triaxial confinement, failure can be triggered if part of the ice is stress free. In this case the behavior of the ice under near-hydrostatic loading will probably depend upon how the loads are applied, gases and liquids giving one result and solid platens giving another.

Although far from complete, it is hoped that this first report will stimulate further interest in this important problem in ice mechanics.

\section{CONGLUSIONS}

From experiments in columnar saline ice loaded under conditions of triaxial confinement at $-10^{\circ} \mathrm{C}$ and a strain rate of $10^{-2} \mathrm{~s}^{-1}$ (i.e. within the brittle regime) it is concluded that:

(i) the maximum principal stresses at failure in the triaxial regime are higher than in the biaxial regime;

(ii) the maximum principal stress at failure appears to be relatively insensitive to the loading path over a large region within the all-compressive octant of principal stress space, at least under the boundary conditions established for metallic-brush platens; and (iii) several mechanisms contribute to brittle compressive failure, each dominating within a particular region of principal stress space.

\section{ACKNOWLEDGEMENTS}

We thank Gary Kuehn and Doug Jones for their help with the testing procedures and discussions of results. This work was sponsored by Office of Naval Research, grant no. N00014-92-J-1279. It was performed at the Ice Research Laboratory which is operated through additional support from Army Research Office, U.S. Coast Guard, Mineral Management Service, Conoco, Exxon, Mobil and MTS Corp.

\section{REFERENCES}

Ashby, M.F. and S.D. Hallam. 1986. The failure of brittle solids containing small cracks under compressive stress states. Acla Metallurgica et Materialia, 34(3), 497-510.

Cannon, N. P., E. M. Schulson, T. R. Smith and H.J. Frost. 1990. Wing cracks and brittle compressive fracture. Acta Metallurgica et Materialia, $38(10), 1955-1962$.

Hallam, S.D. 1986. The role of fracture in limiting ice forces. In International Association for Hydraulic Research. 8th International Symposium on Ice, Iowa City, August 18-22, 1986. Vol. II. Iowa City, IA, University of Iowa. Institute of Hydraulic Research, 287-319.

Häusler, F. U. 1982. Multiaxial compressive strength tests on saline ice with brush-type loading platens. In IAHR. International Symposium on Ice, Québec, Canada, July 27-31, 1981. Proceedings. Vol. II. Québec, Université Laval, 526-536.

Jones, D. E., F. E. Kennedy and E. M. Schulson. 1991. The kinetic friction of saline ice against itself at low sliding velocities. Ann. Glaciol., 15, 242-246.

Jones, S.J. 1982. The confined compressive strength of polycrystalline ice. f. Glaciol., 28(98), 171-177.

Kuehn, G. A. and E. M. Schulson. 1994. The mechanical properties of saline ice under uniaxial compression. Ann. Glaciol., 19 (see paper in this volume).

Kuehn, G. A., R. W. Lee, W. A. Nixon and E. M. Schulson. 1990. The structure and tensile behavior of first-year sea ice and laboratorygrown saline ice. Journal of Offshore Mechanics and Arctic Engineering, 112, $357-363$.

Nemat-Nasser, S. and H. Horii. 1982. Compression-induced nonplanar crack extension with application to splitting, exfoliation, and rockburst. 7. Geophys. Res., 87 (B8), $6805-6821$.

Richter-Menge, J. A. 1991. Confined compressive strength of horizontal first-year sea ice samples. Journal of Offshore Mechanics and Arctic Engineering, 113, 197-207.

Sammonds, P. R., S. A.F. Murrell and M. A. Rist. 1989. Fracture of multi-year sea ice under triaxial stresses: apparatus description and preliminary results. Journal of Offshore Mechanics and Arctic Engineering, 111, 258-263.

Schulson, E. M., G. A. Kuehn, D. E. Jones and D. A. Fifolt. 1991. The growth of wing cracks and the brittle compressive failure of ice. Acta Metallurgica et Materialia, 39(11), 2651-2655.

Smith, T.R. and E. M. Schulson. 1993. The brittle compressive failure of fresh-water columnar ice under biaxial loading. Acta Metallurgica el Materialia, 41 (1), 153-163.

Smith, T.R. and E. M. Schulson. 1994. Brittle compressive failure of salt-water columnar ice under biaxial loading. 7. Glaciol. 40(135), $265-277$.

Timco, G. W. and R. M. W. Frederking. 1986. Confined compression tests: outlining the failure enveloped of columnar sea ice. Cold Reg. Sci. Technol., 12(1), 13-28.

The accuracy of references in the text and in this list is the responsibility of the authors, to whom queries should be addressed. 